\title{
Coding and classification in drug statistics - From national to global application
}

\author{
Marit Rønning \\ WHO Collaborating Centre for Drug Statistics Methodology, P.O. Box 183 Kalbakken, NO-0903 Oslo, Norway \\ Telephone: +4722169810 Telefax:+4722169818 e-mail: marit.ronning@nmd.no
}

\begin{abstract}
SUMMARY
The Anatomical Therapeutic Chemical (ATC) classification system and the defined daily dose (DDD) was developed in Norway in the early seventies. The creation of the ATC/DDD methodology was an important basis for presenting drug utilisation statistics in a sensible way. Norway was in 1977 also the first country to publish national drug utilisation statistics from wholesalers on an annual basis. The combination of these activities in Norway in the seventies made us a pioneer country in the area of drug utilisation research. Over the years, the use of the ATC/DDD methodology has gradually increased in countries outside Norway. Since 1996, the methodology has been recommended by WHO for use in international drug utilisation studies. The WHO Collaborating Centre for Drug Statistics Methodology in Oslo handles the maintenance and development of the ATC/DDD system. The Centre is now responsible for the global co-ordination. After nearly 30 years of experience with ATC/DDD, the methodology has demonstrated its suitability in drug use research. The main challenge in the coming years is to educate the users worldwide in how to use the methodology properly.
\end{abstract}

\section{HISTORY}

In order to measure drug use, it is important to have both a classification system and a unit of measurement. This need was formulated in the late sixties, which still was an infancy period in the field of drug utilisation research. Due to an increasing interest in this field, a symposium entitled The Consumption of Drugs took place in Oslo in 1969 organised by the WHO Regional Office for Europe. It was at this symposium The Drug Utilisation Research Group (DURG) was established tasked with the development of internationally applicable methods for drug utilisation research (1).

Inspired by this interest, a system named the Anatomical Therapeutic Chemical (ATC) classification was developed by the Norwegian Medicinal Depot (NMD). Norwegian researchers also developed a technical unit of measurement called the Defined Daily Dose (DDD) to be used in drug utilisation studies (2). The ATC/DDD system has been used in Norway since the early seventies for presenting drug consumption data.

In 1975, the Nordic Council on Medicines (NLN) was established and collaborated with NMD to further develop the ATC/DDD system. The NLN published the Nordic Statistics on Medicines using the ATC/DDD methodology for the first time in 1979 (3). Since that time the ATC/DDD system has expanded to include most drugs on the Nordic market. At the same time, international interest in the ATC/DDD system for drug utilisation research expanded beyond the Nordic countries largely through the activities of the DURG who recommended the ATC/DDD methodology for international drug utilisation studies.

\section{WHO Collaborating Centre for Drug Statistics Methodology}

In 1981, the WHO Regional Office for Europe decided to recommend the ATC/DDD system for drug utilisation studies in Europe. In connection with this, and to make the methodology more widely used, there was a need for a central body responsible for co-ordinating the use of the methodology. The WHO Collaborating Centre for Drug Statistics Methodology was accordingly established in Oslo in 1982. The Centre is situated at the Norwegian Medicinal Depot (NMD) and has since 1995 been financed by the Norwegian Ministry of Health and Social Affairs.

In 1996, WHO recognised the need to develop use of the ATC/DDD system as an international standard for drug utilisation studies. The Centre was therefore linked directly to WHO Headquarters in Geneva instead of the WHO Regional Office for Europe in Copenhagen. This was seen as important to allow close integration of international drug utilisation studies and WHO's initiatives to achieve universal access to needed drugs and rational use of drugs particularly in developing countries. 
According to the agreement between WHO and the Norwegian government, the Centre's main tasks are development and maintenance of the ATC/DDD system, including:

- To classify drugs according to the ATC system.

- To establish DDDs for drugs which have been assigned an ATC code.

- To review and revise as necessary the ATC classification system and DDDs.

- To stimulate and influence the practical use of the ATC system by co-operating with researchers in the drug utilisation field.

In 1996, when the decision on globalizing the ATC/ DDD system was taken, the WHO Division of Drug Management and Policies in Geneva established the WHO International Working Group for Drug Statistics Methodology. This working group now consists of 12 WHO appointed experts in clinical pharmacology, drug utilisation, drug regulation, drug evaluation, statistics and medicine. The members of the Working Group represent different users of the ATC/DDD system and different nationalities as they represent the 6 WHO global regions. The following countries are represented in the Working Group: Australia, Japan, India, Pakistan, Marocco, Ghana, Zimbabwe, USA, Argentina, The Netherlands and Sweden. The Working Group meets twice annually. The WHO Collaborating Centre for Drug Statistics Methodology receives expert advice from the Working Group and acts as secretariat for the group.

\section{ABOUT THE ATC/DDD SYSTEM}

In the ATC classification system, the drugs are divided into different groups according to the organ or system on which they act and their chemical, pharmacological and therapeutic property (4). Drugs are classified in groups at five different levels. The drugs are divided into fourteen main groups (1st level), with two therapeutic/pharmacological subgroups (2nd and 3rd levels). The 4th level is a therapeutic/pharmacological/ chemical subgroup and the 5th level is the chemical substance. The complete classification of metformin illustrates the structure of the code:

A Alimentary tract and metabolism (1st level, anatomical main group)

A10 Drugs used in diabetes (2nd level, pharmacological/therapeutic main group)

A10B Oral blood glucose lowering drugs (3rd level, chemical/pharmacological/therapeutic subgroup)

A10BA Biguanides (4th level, chemical/pharmacological/therapeutic subgroup)

A10BA02 Metformin (5th level, subgroup for chemical substance)

Thus, in the ATC system all plain metformin preparations are given the code A10BA02.
The basic definition of the DDD is: the assumed average maintenance dose per day for a drug used for its main indication in adults (4). It is important to emphasise that the defined daily dose is a unit of measurement and does not necessarily reflect the recommended or Prescribed Daily Dose. Doses for individual patients and patient groups will often differ from the DDD and will necessarily have to be based on individual characteristics (e.g. age and weight) and pharmacokinetic considerations.

Drug consumption figures should preferably be presented as numbers of DDDs/1000 inhabitants/day or, as DDDs per 100 bed days when in-hospital drug use is considered. Sales or prescription data presented in DDD/1000 inhabitants/day provide a rough estimate of the proportion of the population within a defined area treated daily with certain drugs. For example, the figure $10 \mathrm{DDDs} / 1000$ inhabitants/day indicates that $1 \%$ of the population on average gets a certain treatment daily.

\section{NORWEGIAN DRUG UTILISATION STATISTICS}

Drug utilisation statistics based on wholesale data has been produced in Norway since 1973. In May 1976, a committee was nominated by the Norwegian Board of Health with the task to formulate recommendations for a broader presentation and use of the statistical material compiled at the drug wholesaler, the Norwegian Medicinal Depot (NMD). In December the same year, the recommendations were presented and it was decided that NMD every year should issue a publication containing detailed information on the consumption of drugs in Norway aiming at the medical professions. The first publication was issued in 1977 and included data from the period 1974-1976 (5).

The drug wholesale monopoly in Norway was removed in 1995. It was then decided to continue publishing the wholesale statistics in agreement with and financed by the Ministry of Health and Social Affairs. Data from all the three wholesalers now had to be included.

In 2000, the 22nd edition of the publication "Drug Consumption in Norway" was published covering data from the period 1995-1999 (6). An Internet version of the publication has been available since June 2000 (www.legemiddelforbruk.nmd.no).

Drug statistics from wholesalers are basically relatively precise and offer important tools for medical and health economic evaluations and for the suggestions of appropriate regulatory changes, information activities etc. The statistics can be used to:

- survey consumption, and hence therapy, on different levels and during time

- measure the effect of legislative changes and informational efforts

- define needs for further investigation of drug therapy traditions 
The creation of the ATC/DDD methodology was an important basis for presenting drug utilisation statistics in a sensible way. Norway was one of the first countries to publish national drug utilisation statistics on an annual basis. The combination of these activities in Norway in the seventies made us a pioneer country in the area of drug utilisation research.

\section{COMPARABLE INTERNATIONAL DRUG UTILISATION STATISTICS?}

An important basis for the WHO recommendation to use the ATC/DDD methodology in drug utilisation studies is the possibility of making international comparisons and to study long term trends in drug use. As a result of the rapid increase in the use of the methodology worldwide, it is appropriate to ask how far we have come with regard to comparable international drug utilisation statistics. The answer is not straightforward.

Due to continuous changes and development of the drug assortment and drug uses, a regular revision of the ATC system is necessary. Some ATC and DDD alterations are made annually, and an updated version of the list that includes all officially assigned ATC codes and DDDs is issued every year (7). In order to compare data from different countries and different periods, it is essential to know which ATC codes and DDDs are used. Consumption figures may look very different when different ATC/DDD versions are applied. To illustrate the impact of using different

Fig. 1: Consumption of ACE inhibitors (C09A) in Norway $1987-1999$

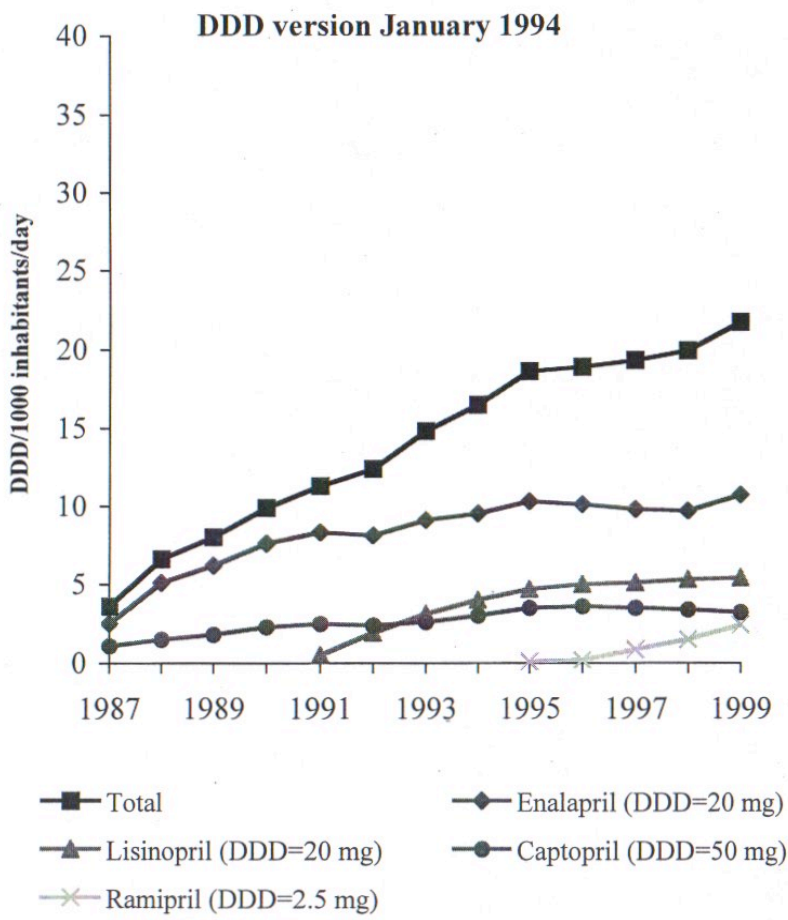

DDD values on drug consumption data, data on the consumption of ACE inhibitors (ATC group C09A) is presented. The DDDs for the ACE inhibitors were revised in 1994, and this revision resulted in a change in the DDD for some of the substances. Figures 1 and 2 show the consumption of ACE inhibitors in Norway in the period 1987-1999 and illustrate that the use of different DDD versions may have a big influence on drug consumption figures. A complete cumulative list of all DDD alterations made is available on the website of the Centre in Oslo (www.whocc.nmd.no).

A literature survey of published drug utilisation studies in the nineties indicated that it is not common practice to give information about the ATC codes and DDD used (8). One possible reason for this might be little or no knowledge about the ATC/DDD system as a dynamic system where alterations are made annually. The lack of references to the actual DDDs used in the studies makes comparisons between different data sets difficult and misleading.

Another important basis for correct and uniform use of the ATC/DDD methodology between countries is the linking of ATC codes and DDDs to each drug package. The link between the national drug register and ATC/DDDs is a job that has to be done by competent persons with proper knowledge of the methodology. Over the years, the WHO Centre's experience has been that in many countries, the health authorities, health policy makers and researchers have not allocated satisfactory resources to this important initial step, and the job has been done locally when needed for a
Fig. 2: Consumption of ACE inhibitors (C09A) in Norway 1987 - 1999

DDD version January 2000

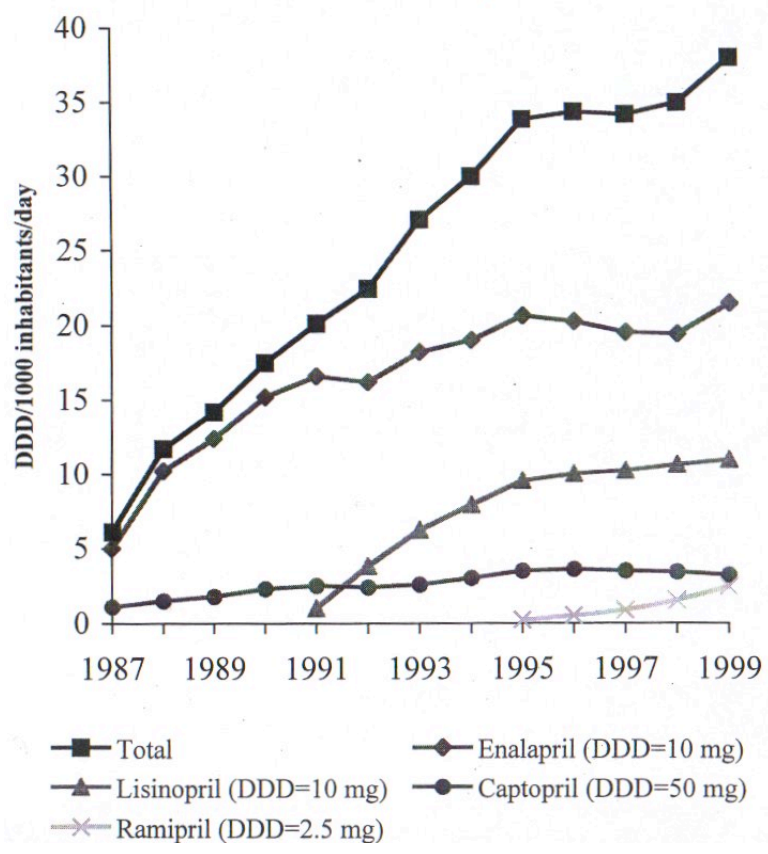


certain research project. The general attitude has been that the ATC classification is ready to use and easy to adopt to the drug products in question. An important criterion for setting up the ATC classification of drugs and the DDD is to realize that the work with allocating ATC codes to the products needs resources and necessary competence. This task should be done on a national basis to secure consistent use of the ATC/DDD methodology within a country. Proper knowledge about the ATC/DDD system and about medicines in general is essential in order to handle this work. The same substance may have different ATC codes depending on the application form and to some extent even the strength. For combination products, specific guidelines are established for allocating ATC codes to the different combinations (4). Allocating DDDs to the products necessitates many of the same considerations. In order to link the drug list with sales figures or prescription figures for preparing drug consumption statistics, it is necessary to make proper calculations such as number of DDDs per drug package. Finally, in a certain country, there will nearly always be medicines and combination products for which no ATC codes or DDDs exists. In these cases, it is important to address the WHO Collaborating Centre for Drug Statistics Methodology in Oslo and request new ATC codes and
DDDs and not create national values. Once ATC codes and DDDs have been linked to the national drug lists, it is necessary to make regular updates in the drug list in accordance with the annual updates of the ATC/ DDD system.

\section{CONCLUSION}

After nearly 30 years of experience with ATC/DDD, the methodology has demonstrated its suitability in drug use research. The rapid increase in the number of users is a good indicator of the usefulness of the system. The main challenge in the coming years is to educate the users worldwide on how to use the methodology properly. The benefits and limitations of the methodology need to be clearly focused. An important criterion for setting up the ATC classification of drugs and the DDD is to realise that the work with allocating ATC codes to the products needs resources and necessary competence. Another important goal is to make the users more aware of the ATC/DDD system as a dynamic system where changes are made annually. When publishing drug utilisation studies, it is very important that proper references are given to the ATC/DDD version used in order to use these studies for international comparisons over time.

\section{REFERENCES}

1. Dukes MNG. Drug utilization studies - Methods and uses. WHO Regional Publications European Series No. 45, Copenhagen, Denmark, 1993: 1-4.

2. Baksaas-Aasen, et al. Drug Dose Statistics, Norsk Medisinaldepot, Oslo, Norway, 1975.

3. The Nordic Council on Medicines. Nordisk Läkemedelsstatistik 1975-1977. Statistical reports of the Nordic Countries no 35, Part 1, Helsingfors, Finland, 1979.

4. Guidelines for ATC classification and DDD Assignment. WHO Collaborating Centre for Drug Statistics Methodology, Oslo, Norway, January 2001.

5. Legemiddelforbruket $i$ Norge (1974-1976). Norsk Medisinaldepot, Oslo, Norway, 1977.

6. Legemiddelforbruket i Norge (Drug Consumption in Norway) 1995-1999. Norsk Medisinaldepot ASA, Oslo, Norway, 2000.

7. ATC Index with DDDs. WHO Collaborating Centre for Drug Statistics Methodology, Oslo, Norway, January 2001.

8. Rønning M, Blix HS, Harbø BT, Strøm H. Different versions of the anatomical therapeutic chemical classification system and the defined daily dose - are drug utilisation data comparable? Eur J Clin Pharmacol 2000; 56: $723-727$. 